\title{
Tensor Fasciae Lata
}

National Cancer Institute

\section{Source}

National Cancer Institute. Tensor Fasciae Lata. NCI Thesaurus. Code C53072.

A muscle of the gluteal region that arises from the iliac crest and inserts into the iliotibial tract of the fascia lata; it functions to stabilize the knee in extension and in hip flexion. 